\title{
Phase-space Symmetry and the Action Function of the Pendulum Problem
}

\author{
S. Olszewski, T. Roliński, M. Baszczak, and R. Kozak \\ Institute of Physical Chemistry of the Polish Academy of Sciences, 44/52 Kasprzaka, \\ 01-224 Warsaw, Poland \\ Reprint requests to S. O.; Fax: 48(22)632-52-76; E-mail: olsz@ichf.edu.pl \\ Z. Naturforsch. 57a, 888-896 (2002); received May 25, 2002
}

\begin{abstract}
An approach to the pendulum problem, which is an alternative to the well-known traditional treatment of that problem, has been formulated. An advantage of the new approach is provided by a full symmetry in the position and momentum variables of the Hamiltonian expression for the energy of the system. A similar symmetry holds for the Hamilton equations describing the motion of a pendulum-like point mass. Calculations of the action function for the two kinds of pendulum Hamiltonians - the traditional one and the new one - are presented.
\end{abstract}

Key words: Pendulum; Phase-space Symmetry; Action Function.

\section{Introduction. Traditional Treatment of the Phase space of a Pendulum}

The mathematical pendulum and its phase space is still of vivid interest $[1-6]$. The dynamic moment invariants have been investigated for linear Hamiltonian systems and a non-linear Hamiltonian system like that of a pendulum in [7] and [8], respectively. But a thorough examination of the action function of a pendulum considered as an adiabatic invariant of a dynamical system (see e.g. [9]) seems to be lacking. In general, the calculation of the action function is important because its properties are strictly related to the distribution of the position-momentum variables possessed by the pendulum point mass in the phase space.

The aim of the present paper is to present a new Hamiltonian and the corresponding new set of the Hamilton equations for the motion of a pendulum. These equations have the advantage of being easily integrated in the form of Fourier expansions. Another basic feature of this new set of equations of motion is their full symmetry in the position-momentum coordinates. This property enables an easily calculation of the action function and gives a full symmetry of the phase-space diagrams plotted for some constant energy. Furthermore, the action function provided by the new Hamiltonian can be easily referred to that of the traditional Hamiltonian. So both action functions can readily be calculated. We give an outline of the traditional theory before the presentation of the new approach to the pendulum equations.
Usually the description of a pendulum, which is a point-like mass $m$ oscillating planarly at some constant distance $l$ from a fixed point, is done with the angular momentum $p_{\varphi}$ and the position angle $\varphi$, the latter being the angle of deviation from the position of the pendulum at rest [9-14]. We have

$$
p_{\varphi}=m^{2} \dot{\varphi},
$$

and the Hamiltonian of the problem is

$$
\begin{aligned}
H\left(\varphi, p_{\varphi}\right) & =\frac{p_{\varphi}^{2}}{2 m l^{2}}+m g l(1-\cos \varphi) \\
& =\frac{m}{2} l^{2} \dot{\varphi}^{2}+m g l(1-\cos \varphi) ;
\end{aligned}
$$

$g$ is the acceleration constant of gravity. One of the Hamilton equations, viz.

$$
\dot{p}_{\varphi}=m l^{2} \ddot{\varphi}=-\frac{\partial H}{\partial \varphi}=-m g l \sin \varphi,
$$

leads to the fundamental equation of the pendulum motion

$$
\ddot{\varphi}=-\frac{g}{l} \sin \varphi
$$

whereas the other Hamilton equation, namely

$$
\dot{\varphi}=\frac{\partial H}{\partial p_{\varphi}}=\frac{p_{\varphi}}{m l^{2}},
$$

is equivalent to equation (1). 
In principle, (4) attains its readily solvable form only for small $\varphi$, equivalent to the harmonic oscillator equation for $\varphi$. For larger $\varphi$ the solution of (4) becomes a much more complicated task.

We can assume that the motion of the pendulum begins at $\varphi=\varphi_{0}$, where

$$
\dot{\varphi}\left(\varphi_{0}\right)=\dot{\varphi}_{0}=0 .
$$

Because of the conservation of the pendulum energy $E^{\left(\varphi, p_{\varphi}\right)}$, we have from (2) and (5)

$$
E^{\left(\varphi, p_{\varphi}\right)}=1-\cos \varphi_{0}=2 \sin ^{2}\left(\varphi_{0} / 2\right),
$$

where, for simplicity we have put

$$
m=g=l=1 \text {. }
$$

With (7) the pendulum oscillation frequency $\omega$ for small $\varphi$ takes the form

$$
\omega_{0}=\left(\frac{g}{l}\right)^{1 / 2}=1
$$

Basing on (6), the pendulum energy is parameterized by the variable $\varphi_{0}$. From (2) and the abbreviations in (7) we have

$$
1-\cos \varphi_{0}=\frac{1}{2}\left(\frac{\mathrm{d} \varphi}{\mathrm{d} t}\right)^{2}+1-\cos \varphi
$$

so

$$
\mathrm{d} t=\frac{\mathrm{d} \varphi}{2^{1 / 2}\left(\cos \varphi-\cos \varphi_{0}\right)^{1 / 2}} .
$$

With the aid of substitutions

$$
\sin \left(\varphi_{0} / 2\right)=k
$$

and

$$
\sin (\varphi / 2)=\left(\frac{1}{2}\right)^{1 / 2}(1-\cos \varphi)^{1 / 2}=k \sin z,
$$

(10) can be integrated giving the well-known combination of elliptic functions

$t=\int_{z}^{\pi / 2} \frac{\mathrm{d} z}{\left(1-k^{2} \sin ^{2} z\right)^{1 / 2}}=F(k, \pi / 2)-F(k, z)$.
The whole time period of the pendulum oscillation is represented by the formula $[9,12,14]$

$$
\begin{aligned}
T= & \frac{4}{\sqrt{2}} \int_{0}^{\varphi_{0}} \frac{\mathrm{d} \varphi}{\left(\cos \varphi-\cos \varphi_{0}\right)^{1 / 2}} \\
= & 4 K\left[\sin \left(\varphi_{0} / 2\right)\right] \\
= & 2 \pi\left[1+\frac{1^{2}}{2^{2}} \sin ^{2}\left(\varphi_{0} / 2\right)+\frac{1^{2} 3^{2}}{2^{2} 4^{2}} \sin ^{4}\left(\varphi_{0} / 2\right)\right. \\
& \left.+\frac{1^{2} 3^{2} 5^{2}}{2^{2} 4^{2} 6^{2}} \sin ^{6}\left(\varphi_{0} / 2\right)+\ldots\right] .
\end{aligned}
$$

On the other hand, the time period of the pendulum having the frequency $\omega_{0}$ given in (8) is

$$
T_{0}=2 \pi \text {. }
$$

The phase diagram for a pendulum is usually given by a plot of the variable $2^{1 / 2} \dot{\varphi}$ represented as a function of $\varphi[12-14]$. This plot can be done for different values of $\dot{\varphi}_{0}$ and $\varphi_{0}$. Since in our case we assume $\dot{\varphi}_{0}=0$, see (5), the diagram obtained from (9a) is given by a plot of the expression

$$
\frac{\dot{\varphi}(\varphi)}{\sqrt{2}}= \pm \sqrt{\cos \varphi-\cos \varphi_{0}}
$$

considered as a function of the variable $\varphi$. Different values of $\varphi_{0}$ entering (14) are taken from the interval

$$
0<\varphi_{0}<\pi
$$

\section{A Pendulum Hamiltonian being Symmetrical in Position-momentum Coordinates}

Evidently, $H\left(\varphi, p_{\varphi}\right)$ in (2) is not symmetrical in the variables $\varphi, p_{\varphi}$, and our first aim is to transform the Hamiltonian (2) into a Hamiltonian $H\left(x, p_{x}\right)$ dependent symmetrically on $x$ and $p_{x}$; here $x$ and $p_{x}$ are Cartesian coordinates of position and momentum, respectively. This symmetrical Hamiltonian is postulated to have the form

$$
H\left(x, p_{x}\right)=1-\cos x \cos p_{x}=E^{\left(x, p_{x}\right)},
$$


where the energy $E^{\left(x, p_{x}\right)}$ is a constant independent of $x$ and $p_{x}$. A characteristic property of (16) is that for small $x$ and $p_{x}$ the expression $H\left(x, p_{x}\right)$ takes the form

$$
H\left(x, p_{x}\right) \cong \frac{p_{x}^{2}}{2}+\frac{x^{2}}{2}
$$

which is a typical harmonic-oscillator Hamiltonian.

Equations following from the Hamiltonian (16) are

$$
\begin{aligned}
& \dot{x}=\frac{\partial H\left(x, p_{x}\right)}{\partial p_{x}}=\cos x \sin p_{x}, \\
& \dot{p}_{x}=-\frac{\partial H\left(x, p_{x}\right)}{\partial x}=-\sin x \cos p_{x},
\end{aligned}
$$

but the next time-differentiation of (17) gives

$$
\begin{aligned}
\ddot{x} & \equiv \frac{\mathrm{d} \dot{x}}{\mathrm{~d} t}=\frac{\mathrm{d}\left(\cos x \sin p_{x}\right)}{\mathrm{d} t} \\
& =-\sin x \cos x \sin ^{2} p_{x}+\cos ^{2} p_{x}(-\sin x) \cos x \\
& =-\frac{1}{2} \sin 2 x\left(\sin ^{2} p_{x}+\cos ^{2} p_{x}\right)=-\frac{1}{2} \sin 2 x,
\end{aligned}
$$

or

$$
\frac{\mathrm{d}^{2}}{\mathrm{~d} t^{2}}(2 x)=-\sin (2 x)
$$

A differential equation similar to that for $x$ can be derived also for $p_{x}$. This becomes evident from the time differentiation of (17a):

$$
\begin{aligned}
\ddot{p}_{x} & =-\cos x \cos p_{x} \dot{x}+\sin x \sin p_{x} \dot{p}_{x} \\
& =-\cos ^{2} x \cos p_{x} \sin p_{x}-\sin ^{2} x \sin p_{x} \cos p_{x} \\
& =-\cos p_{x} \sin p_{x}=-\frac{1}{2} \sin \left(2 p_{x}\right) .
\end{aligned}
$$

In the second step of (18b), (17) and (17a) are applied.

Equations (18a) and (18b) are typical pendulumlike formulae. They can be solved on the basis of Fourier expansions in the form

$$
\begin{aligned}
& x=a_{1} \cos \omega t+a_{3} \cos 3 \omega t+a_{5} \cos 5 \omega t+\ldots, \\
& p_{x}=b_{1} \sin \omega t+b_{3} \sin 3 \omega t+b_{5} \sin 5 \omega t+\ldots
\end{aligned}
$$

Here an opposite boundary condition for $p_{x}$ to that assumed for $x$, which is

$$
x(t=0)=a_{0},
$$

should be taken into account; see (21) below. The procedure of solution done with the aid of the smallparameter method [15] provides us also with $\omega$; the details of the Fourier expansions - together with $\omega-$ are presented in Table 1 . One of the properties of $a_{i}$ and $b_{i}$ entering (19) and (19a) is that

$$
b_{i}=(-1)^{\frac{1}{2}(i+1)} a_{i},
$$

and another property is that

$$
a_{1}+a_{3}+a_{5}+\ldots=a_{0},
$$

which is a consequence of (19) and (19b).

Evidently, on the basis of (7), (18a) is identical with (4) if we put

$$
\varphi=2 x
$$

In the next step, because of (1), (2), (7) and (20), we obtain

$$
\begin{aligned}
H\left(\varphi, p_{\varphi}\right) & =\frac{1}{2} p_{\varphi}^{2}+1-\cos \varphi \\
& =\frac{1}{2} \dot{\varphi}^{2}+1-\cos \varphi \\
& =2 \dot{x}^{2}+1-\cos (2 x) \\
& =2\left(\dot{x}^{2}+\sin ^{2} x\right)=E^{\left(\varphi, p_{\varphi}\right)} .
\end{aligned}
$$

This formula is rather different form the Hamiltonian (16), which has the advantage of symmetry in the coordinates $x$ and $p_{x}$.

The boundary conditions for $p_{x}$ are

$$
p_{x}=0 \text { at } x=x_{0}=a_{0},
$$

where $a_{0}$, the largest value attained by $x$, is called the amplitude of the motion of the pendulum particle. From the property of symmetry of $H$ in the variables $x$ and $p_{x}$ we have also

$$
p_{x}=a_{0} \text { at } x=0 \text {; }
$$


Table 1. Fourier-like solutions of (18a) and (18b).

$$
\begin{aligned}
x(t)= & \cos (\omega t)\left(a_{0}+\frac{1}{48} a_{0}^{3}+\frac{17}{3840} a_{0}^{5}+\frac{1487}{1290240} a_{0}^{7}+\frac{30901}{743178240} a_{0}^{9}+\ldots\right) \\
& +\cos (3 \omega t)\left(-\frac{1}{48} a_{0}^{3}-\frac{1}{192} a_{0}^{5}-\frac{133}{92160} a_{0}^{7}-\frac{16027}{37158912} a_{0}^{9}+\ldots\right)+\cos (5 \omega t)\left(\frac{1}{1280} a_{0}^{5}+\frac{1}{3072} a_{0}^{7}+\frac{1211}{10321920} a_{0}^{9}+\ldots\right) \\
& +\cos (7 \omega t)\left(-\frac{1}{28672} a_{0}^{7}-\frac{1}{49152} a_{0}^{9}+\ldots\right)+\cos (9 \omega t)\left(\frac{1}{589824} a_{0}^{9}+\ldots\right)+\ldots \\
p_{x}(t)= & \sin (\omega t)\left(-a_{0}-\frac{1}{48} a_{0}^{3}-\frac{17}{3840} a_{0}^{5}-\frac{1487}{1290240} a_{0}^{7}-\frac{30901}{743178240} a_{0}^{9}-\ldots\right) \\
& +\sin (3 \omega t)\left(-\frac{1}{48} a_{0}^{3}-\frac{1}{192} a_{0}^{5}-\frac{133}{92160} a_{0}^{7}-\frac{16027}{37158912} a_{0}^{9}+\ldots\right)+\sin (5 \omega t)\left(-\frac{1}{1280} a_{0}^{5}-\frac{1}{3072} a_{0}^{7}-\frac{1211}{10321920} a_{0}^{9}-\ldots\right) \\
& +\sin (7 \omega t)\left(-\frac{1}{28672} a_{0}^{7}-\frac{1}{49152} a_{0}^{9}+\ldots\right)+\sin (9 \omega t)\left(-\frac{1}{589824} a_{0}^{9}-\ldots\right)+\ldots \\
\omega=1 & \frac{1}{4} a_{0}^{2}+\frac{1}{192} a_{0}^{4}-\frac{23}{11520} a_{0}^{6}-\frac{2519}{5160960} a_{0}^{8}+\ldots
\end{aligned}
$$

in this case, $a_{0}$ is a maximum value (amplitude) of the momentum. In both cases, represented by (21) and (21a), the Hamiltonian attains the same value

$$
H\left(x, p_{x}\right)=1-\cos a_{0}=E^{\left(x, p_{x}\right)} .
$$

Evidently, (22) is the energy of system in course of the whole oscillatory motion because of the energy conservation.

The energy (22) can be readily referred to the energy of (20a). For, from (17a)

$$
\dot{x}^{2}=\cos ^{2} x \sin ^{2} p_{x}=\cos ^{2} x\left(1-\cos ^{2} p_{x}\right),
$$

and from (16) and (22):

$$
\cos x \cos p_{x}=\cos a_{0} .
$$

In effect, from (23):

$$
\dot{x}^{2}=\cos ^{2} x-\cos ^{2} a_{0}
$$

This result, when substituted into (20a), gives on the basis is of (22)

$$
\begin{aligned}
H\left(\varphi, p_{\varphi}\right) & =2\left(\cos ^{2} x-\cos ^{2} a_{0}+\sin ^{2} x\right) \\
& =2 \sin ^{2} a_{0}=2\left(1+\cos a_{0}\right) H\left(x, p_{x}\right) .
\end{aligned}
$$

The second step obtained in (25) remains in accordance with (6), provided that we take into account (20) at $\varphi=\varphi_{0}$ and (21); let us note that $\dot{\varphi}_{0}=0$ is coincident with the condition that $p_{x}=0$. We summarize Section 2 by stating that the pendulum motion considered in $\left(\varphi, p_{\varphi}\right)$-coordinates can be replaced by a similar one-dimensional oscillatory motion in $\left(x, p_{x}\right)$-coordinates, but $x$ and $p_{x}$ build up a symmetrical position-momentum Hamiltonian $H\left(x, p_{x}\right)$. The ratio of the Hamiltonians,

$$
\frac{H\left(\varphi, p_{\varphi}\right)}{H\left(x, p_{x}\right)}=2\left(1+\cos a_{0}\right),
$$

remains a constant number in the course of the whole oscillatory motion depending solely on the amplitude $a_{0}=\varphi_{0} / 2$.

\section{Symmetrical Phase Diagram for a Pendulum and its Properties}

The phase diagram in the space of $\left(x, p_{x}\right)$ can be readily obtained when $p_{x}$ of $(18 \mathrm{~b})$ is plotted as a function of $x$ taken from (18), or (18a). In Section 3 we examine the general properties of that plot which is represented by a closed curve on the condition that the curvature 


$$
\chi=\frac{\frac{\mathrm{d}^{2} p_{x}}{\mathrm{~d} x^{2}}}{\left[1+\left(\frac{\mathrm{d} p_{x}}{\mathrm{~d} x}\right)^{2}\right]^{3 / 2}}
$$

remains a positive number. Since from (16) we have

$$
\cos p_{x}=\frac{1-E^{\left(x, p_{x}\right)}}{\cos x},
$$

the curvature becomes

$$
\chi=\frac{\left[1-E^{\left(x, p_{x}\right)}\right]\left(\sin ^{2} x+\sin ^{2} p_{x}\right)}{\left(\cos ^{2} x \sin ^{2} p_{x}+\sin ^{2} x \cos ^{2} p_{x}\right)^{-3 / 2}}
$$

By taking solely into account that $E^{\left(x, p_{x}\right)}>0$ we see that $\chi$ is a positive number on the condition that

$$
0<E^{\left(x, p_{x}\right)}<1 .
$$

Phase diagrams of $p_{x}$ versus $x$, plotted for different $E^{\left(x, p_{x}\right)}$, are presented in Figure 1.

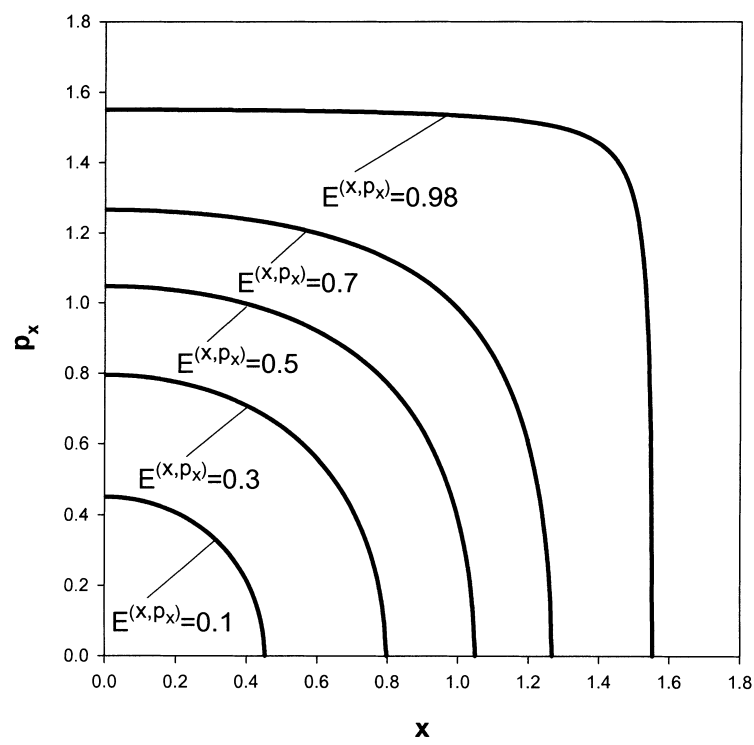

Fig. 1. One-fourth of the phase diagrams of $p_{x}$ versus $x$ calculated from solutions of (18a) and (18b) for different $E^{\left(x, p_{x}\right)}$ of (16) (full diagrams are symmetrical to the axes $x$ and $p_{x}$ ). For details of $x$ and $p_{x}$ see Table 1 .

\section{Action Functions for a Pendulum and their Properties}

Since the calculation of the action function for the pendulum problem, described with the aid of the Hamiltonian $H\left(\varphi, p_{\varphi}\right)$, seems to be rather difficult [9], we begin with the calculation of a similar action function for the Hamiltonian $H\left(x, p_{x}\right)$. We have

$$
J^{\left(x, p_{x}\right)}=\oint p_{x} \mathrm{~d} x
$$

$$
=\int_{0}^{T} p_{x} \frac{\mathrm{d} x}{\mathrm{~d} t} \mathrm{~d} t=-\int_{0}^{T} x \frac{\mathrm{d} p_{x}}{\mathrm{~d} t} \mathrm{~d} t
$$

This integral, calculated on the basis of $x=x(t)$ and $p_{x}=p_{x}(t)$ given in Table 1 , is presented in Table $2 ; T$ is the time period of the pendulum oscillation, see (13).

The validity of the result obtained for $J^{\left(x, p_{x}\right)}$ can be checked when this function - together with the energy (22) - is applied to the calculation of the frequency:

$\omega=\frac{2 \pi}{T}=\left[\frac{\partial J^{\left(x, p_{x}\right)}\left(a_{0}\right)}{\partial E^{\left(x, p_{x}\right)}\left(a_{0}\right)}\right]^{-1}=\left[\frac{\partial J^{\left(x, p_{x}\right)} / \partial a_{0}}{\partial E^{\left(x, p_{x}\right)} / \partial a_{0}}\right]^{-1}$.

Equation (30) holds, in fact, on condition we put in (13)

$$
\frac{\varphi_{0}}{2}=a_{0}
$$

see (20) and (21). But the same $\omega$ should be obtained also on the basis of the expression $H\left(\varphi, p_{\varphi}\right)$ and the

Table 2. Action functions $J$ (and their derivatives) calculated in terms of the power series of the amplitude $a_{0}$. A reference between two kinds of derivatives of $J$ descending, respectively, from two kinds of the Hamiltonians, is given in $(32 \mathrm{a})$.

$$
\begin{aligned}
& J^{\left(x, p_{x}\right)}=2 \pi\left(\frac{1}{2} a_{0}^{2}+\frac{1}{48} a_{0}^{4}+\frac{23}{5760} a_{0}^{6}+\frac{593}{645120} a_{0}^{8}+\ldots\right) . \\
& \frac{\partial J^{\left(x, p_{x}\right)}}{\partial a_{0}}=2 \pi\left(a_{0}+\frac{1}{12} a_{0}^{3}+\frac{23}{960} a_{0}^{5}+\frac{593}{80640} a_{0}^{7}+\ldots\right) . \\
& J^{\left(\varphi, p_{\varphi}\right)}=8 \pi\left(\frac{1}{2} a_{0}^{2}-\frac{5}{48} a_{0}^{4}+\frac{23}{5760} a_{0}^{6}-\frac{41}{129024} a_{0}^{8}+\ldots\right) .
\end{aligned}
$$

$\frac{\partial J^{\left(\varphi, p_{\varphi}\right)}}{\partial a_{0}}=8 \pi\left(a_{0}-\frac{5}{12} a_{0}^{3}+\frac{23}{960} a_{0}^{5}-\frac{41}{16128} a_{0}^{7}+\ldots\right)$. 
corresponding action function $J^{\left(\varphi, p_{\varphi}\right)}$, on the condition that $H\left(x, p_{x}\right)$ in (30) is replaced by $H\left(\varphi, p_{\varphi}\right)$ and the action function $J^{\left(x, p_{x}\right)}$ is replaced by $J^{\left(\varphi, p_{\varphi}\right)}$. Therefore we have the relation

$$
\frac{\frac{\partial H\left(x, p_{x}\right)}{\partial a_{0}}}{\frac{\partial \boldsymbol{J}^{\left(x, p_{x}\right)}}{\partial a_{0}}}=\frac{\frac{\partial H\left(\varphi, p_{\varphi}\right)}{\partial a_{0}}}{\frac{\partial \boldsymbol{J}^{\left(\varphi, p_{\varphi}\right)}}{\partial a_{0}}}
$$

from which we obtain

$$
\frac{\partial J^{\left(\varphi, p_{\varphi}\right)}}{\partial a_{0}}=\frac{\partial J^{\left(x, p_{x}\right)}}{\partial a_{0}} \frac{\frac{\partial H\left(\varphi, p_{\varphi}\right)}{\partial a_{0}}}{\frac{\partial H\left(x, p_{x}\right)}{\partial a_{0}}}=4 \cos a_{0} \frac{\partial J^{\left(x, p_{x}\right)}}{\partial a_{0}}
$$

The right-hand side of (32a), which is easy to calculate on the basis of (22), (25) and $J^{\left(x, p_{x}\right)}$ from Table 2, can be readily integrated over the parameter $a_{0}$ giving $J^{\left(\varphi, p_{\varphi}\right)}$ as a function of $a_{0}$; see Table 2 . The plots of the functions $J^{\left(x, p_{x}\right)}$ and $J^{\left(\varphi, p_{\varphi}\right)}$, done versus $a_{0}$, are compared in Figure 2.

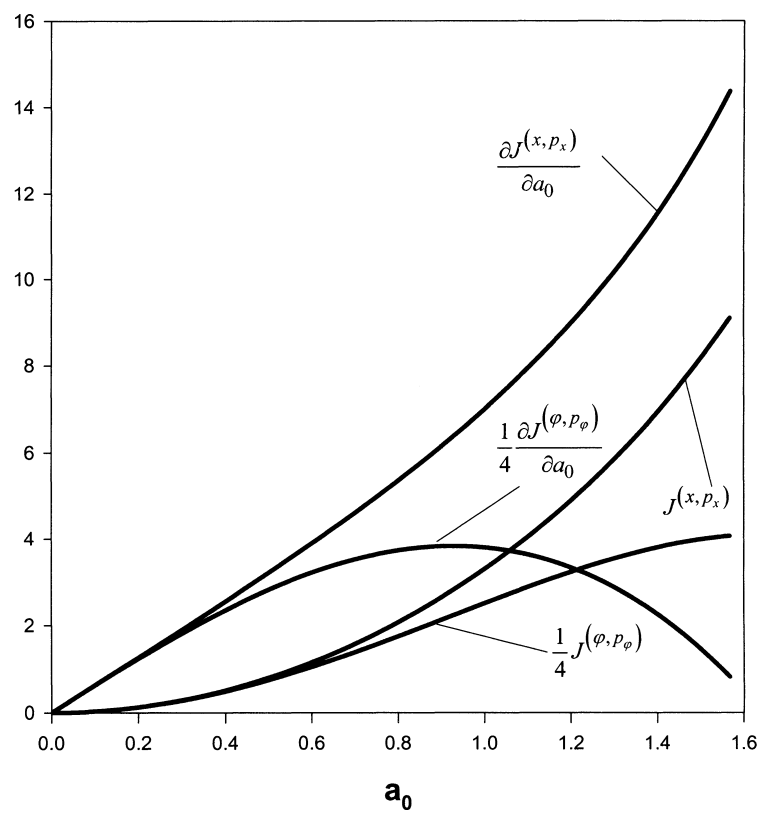

Fig. 2. Plots of the action functions $J^{\left(x, p_{x}\right)}$ and $\frac{1}{4} J^{\left(\varphi, p_{\varphi}\right)}$ compared for two kinds of the pendulum Hamiltonians: $H\left(x, p_{x}\right)$ and $H\left(\varphi, p_{\varphi}\right)$

\section{Verification of some Results of Section 4}

The validity of the result obtained in (32a) and Table 2 can be verified: From relations (6) and (9) we have

$$
\begin{aligned}
\frac{\dot{\varphi}^{2}}{2} & =E^{\left(\varphi, p_{\varphi}\right)}-1+\cos \varphi=E^{\left(\varphi, p_{\varphi}\right)}-2 \sin ^{2} \frac{\varphi}{2} \\
& =2\left[\sin ^{2}\left(\frac{\varphi_{0}}{2}\right)-\sin ^{2}\left(\frac{\varphi}{2}\right)\right],
\end{aligned}
$$

therefore

$$
\begin{aligned}
J^{\left(\varphi, p_{\varphi}\right)} & =\oint p_{\varphi} \mathrm{d} \varphi=\oint \dot{\varphi} \mathrm{d} \varphi \\
& =2^{1 / 2} \oint\left(E^{\left(\varphi, p_{\varphi}\right)}-2 \sin ^{2} \frac{\varphi}{2}\right)^{1 / 2} \mathrm{~d} \varphi \\
& =2^{5 / 2} \int_{0}^{\varphi_{0}}\left(E^{\left(\varphi, p_{\varphi}\right)}-2 \sin ^{2} \frac{\varphi}{2}\right)^{1 / 2} \mathrm{~d} \varphi \\
& =2^{7 / 2} \int_{0}^{u_{0}}\left(2 \sin ^{2} u_{0}-2 \sin ^{2} u\right)^{1 / 2} \mathrm{~d} u \\
& =2^{4} \sin u_{0} \int_{0}^{u_{0}}\left(1-\frac{\sin ^{2} u}{\sin ^{2} u_{0}}\right)^{1 / 2} \mathrm{~d} u
\end{aligned}
$$

Here we have put $\varphi / 2=u, \varphi_{0} / 2=u_{0}$ and $E^{\left(\varphi, p_{\varphi}\right)}=2 \sin ^{2} u_{0}$ because of (33). In the next step we put

$$
\frac{\sin u}{\sin u_{0}}=z, \quad \frac{\cos u}{\cos u_{0}} \mathrm{~d} u=\mathrm{d} z
$$

Since from (35) follows

$$
\cos u=\left(1-\sin ^{2} u_{0} z^{2}\right)^{1 / 2},
$$

we obtain for the integral $J^{\left(\varphi, p_{\varphi}\right)}$

$$
\begin{aligned}
J^{\left(\varphi, p_{\varphi}\right)} & =2^{4} \sin u_{0} \int_{0}^{1} \frac{\left(1-z^{2}\right)^{1 / 2} \mathrm{~d} z}{\left(1-\sin ^{2} u_{0} z^{2}\right)^{1 / 2}}=2^{4} \sin u_{0} \int_{0}^{1} \frac{\left(1-z^{2}\right)^{1 / 2} \mathrm{~d} z}{\frac{1}{\sin ^{2} u_{0}}-z^{2}} \\
& =2^{4}\left[-\cos ^{2} u_{0} F\left(\frac{\pi}{2}, \sin u_{0}\right)+E\left(\frac{\pi}{2}, \sin u_{0}\right)\right] \cdot
\end{aligned}
$$


The functions $F\left(\frac{\pi}{2}, \sin u_{0}\right) \equiv K\left(\sin u_{0}\right) \quad$ and $E\left(\frac{\pi}{2}, \sin u_{0}\right) \equiv E\left(\sin u_{0}\right)$ are the elliptic integrals of the first kind and the second kind, respectively [16].

A derivative of the expression (36) calculated with respect of the variable

$$
u_{0}=a_{0}
$$

[see (31)] gives [17]

$$
\begin{aligned}
\frac{\partial J^{\left(\varphi, p_{\varphi}\right)}}{\partial a_{0}}= & 2^{4}\left\{\sin \left(2 a_{0}\right) K\left(\sin a_{0}\right)\right. \\
& -\cos ^{2} a_{0} \operatorname{ctg} a_{0}\left[\frac{E\left(\sin a_{0}\right)}{\cos ^{2} a_{0}}-K\left(\sin a_{0}\right)\right] \\
& \left.+\operatorname{ctg} a_{0}\left[E\left(\sin a_{0}\right)-K\left(\sin a_{0}\right)\right]\right\} \\
= & 2^{4}\left\{\sin \left(2 a_{0}\right) K\left(\sin a_{0}\right)+\cos ^{2} a_{0} \operatorname{ctg} a_{0}\right. \\
& \left.\times K\left(\sin a_{0}\right)-\operatorname{ctg} a_{0} K\left(\sin a_{0}\right)\right\} \\
= & 2^{4} \sin a_{0} \cos a_{0} K\left(\sin a_{0}\right) .
\end{aligned}
$$

Evidently, this expression tends to zero both at $a_{0}=0$ and $a_{0}=\pi / 2$.

Our idea is now to apply (32a) and (38) to the calculation of the function $\frac{\partial J^{\left(x, p_{x}\right)}}{\partial a_{0}}$. In the first step we demonstrate that a fundamental relation for $\omega^{-1}$, being a reciprocal formula to that presented in (30) for the case of the variables $x$ and $p_{x}$, holds also for the case of $\varphi$ and $p_{\varphi}$ [see (32)]:

$$
\begin{aligned}
2 \pi \omega^{-1} & =\frac{\partial J^{\left(\varphi, p_{\varphi}\right)}}{\partial E^{\left(\varphi, p_{\varphi}\right)}}=\frac{\frac{\partial J^{\left(\varphi, p_{\varphi}\right)}}{\partial a_{0}}}{\frac{\partial E^{\left(\varphi, p_{\varphi}\right)}}{\partial a_{0}}} \\
& =\frac{2^{4} \sin a_{0} \cos a_{0} K\left(\sin a_{0}\right)}{2^{2} \sin a_{0} \cos a_{0}} \\
& =2^{2} K\left(\sin a_{0}\right)=T .
\end{aligned}
$$

This result is obtained on the basis of (38), (31), (25) and (13). In the next step, because of the relation [see (22) and (25)]

$$
\begin{aligned}
\frac{\frac{\partial E^{\left(x, p_{x}\right)}}{\partial a_{0}}}{\frac{\partial E^{\left(\varphi, p_{\varphi}\right)}}{\partial a_{0}}} & =\frac{\frac{\partial\left(1-\cos a_{0}\right)}{\partial a_{0}}}{2 \frac{\partial}{\partial a_{0}}\left(1-\cos ^{2} a_{0}\right)} \\
& =\frac{\sin a_{0}}{2^{2} \cos a_{0} \sin a_{0}}=\frac{1}{4 \cos a_{0}},
\end{aligned}
$$

we have from (32a), (38) and (40)

$$
\frac{\partial J^{\left(x, p_{x}\right)}}{\partial a_{0}}=\frac{\frac{\partial J^{\left(\varphi, p_{\varphi}\right)}}{\partial a_{0}}}{\frac{\partial E^{\left(\varphi, p_{\varphi}\right)}}{\partial a_{0}}} \frac{\partial E^{\left(x, p_{x}\right)}}{\partial a_{0}}=4 K\left(\sin a_{0}\right) \sin a_{0} .
$$

The same expansion can be readily obtained from the derivative of the $a_{0}$-dependent expression calculated for $J^{\left(x, p_{x}\right)}$ in (29); see Table 2.

\section{The Non-linearity Parameter for the Pendulum and the Change of $T$ with the Pendulum Energy}

The non-linearity parameter $\alpha$ for the pendulum can be calculated on the basis of expressions depending on $a_{0}=\varphi_{0} / 2$ and the fundamental formula

$$
\alpha=\frac{J}{\omega} \frac{\mathrm{d} \omega}{\mathrm{d} J}=\frac{J\left(a_{0}\right) \partial \omega / \partial a_{0}}{\omega\left(a_{0}\right) \partial J / \partial a_{0}} .
$$

This expansion, absent in numerous text-books, is presented for the case of $J=J^{\left(\varphi, p_{\varphi}\right)}$ and $J=J^{\left(x, p_{x}\right)}$ in Table 3; the plots of both $\alpha$ 's, taken as functions of $a_{0}$, are given in Figure 3.

A difference existing between the two kinds of the energy expression, $E^{\left(\varphi, p_{\varphi}\right)}$ and $E^{\left(x, p_{x}\right)}$, implies a difference in the behaviour of the change of the oscillation period $T$ attained with the change of energy of the oscillating system. Because of (13), (25) and (31) we have

$$
\begin{aligned}
\frac{\partial T}{\partial E^{\left(\varphi, p_{\varphi}\right)}}= & \frac{\partial T / \partial\left(\varphi_{0} / 2\right)}{\partial E^{\left(\varphi, p_{\varphi}\right)} / \partial\left(\varphi_{0} / 2\right)}=\frac{\partial T / \partial a_{0}}{\partial E^{\left(\varphi, p_{\varphi}\right)} / \partial a_{0}} \\
= & \frac{\pi}{2}\left[\frac{1^{2}}{2}+\frac{1^{2} 3^{2}}{2^{2} 4} \sin ^{2} a_{0}+\frac{1^{2} 3^{2} 5^{2}}{2^{2} 4^{2} 6} \sin ^{4} a_{0}\right. \\
& \left.+\frac{1^{2} 3^{2} 5^{2} 7^{2}}{2^{2} 4^{2} 6^{2} 8} \sin ^{6} a_{0}+\ldots\right]
\end{aligned}
$$


Table 3. Non-linearity parameter $\alpha$ for two kinds of the pendulum Hamiltonian, calculated in terms of the power series of the parameter $a_{0}$; see (42) and inferences given below that formula.

$\alpha^{\left(x, p_{x}\right)}=-\frac{1}{4} a_{0}^{2}-\frac{1}{24} a_{0}^{4}-\frac{143}{11520} a_{0}^{6}-\frac{1403}{322560} a_{0}^{8}+\ldots$

$\alpha^{\left(\varphi \cdot p_{\varphi}\right)}=-\frac{1}{4} a_{0}^{2}-\frac{5}{48} a_{0}^{4}-\frac{533}{11520} a_{0}^{6}-\frac{2657}{129024} a_{0}^{8}+\ldots$

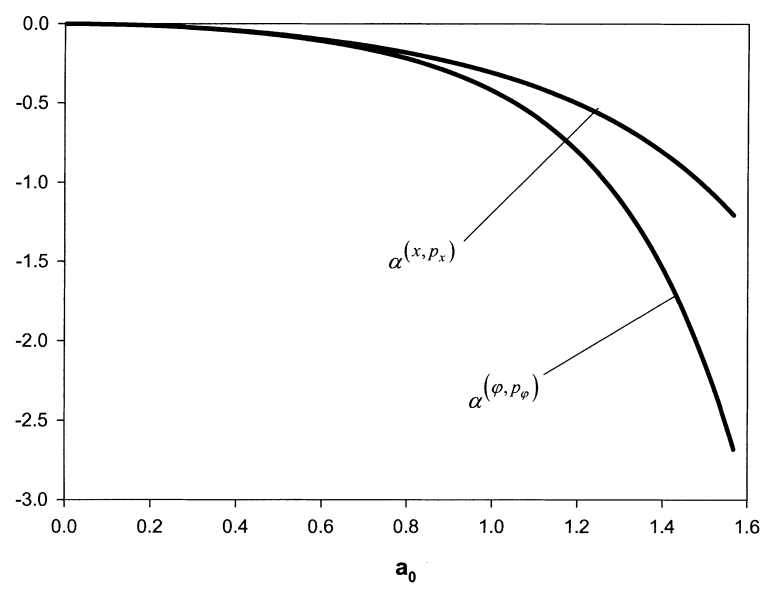

Fig. 3. The non-linearity parameter $\alpha$ [see (42)] plotted versus amplitude $a_{0}$ for two kinds of the pendulum Hamiltonian: $H\left(\varphi, p_{\varphi}\right)$ from (2) and $H\left(x, p_{x}\right)$ from (16).

whereas

$\frac{\partial T}{\partial E^{\left(x, p_{x}\right)}}=\frac{\partial T / \partial a_{0}}{\partial E^{\left(x, p_{x}\right)} / \partial a_{0}}=4 \cos a_{0} \frac{\partial T}{\partial E^{\left(\varphi, p_{\varphi}\right)}}$

Equation (43a) holds because of the relation

$$
\frac{\partial E^{\left(\varphi, p_{\varphi}\right)}}{\partial a_{0}}=4 \cos a_{0} \frac{\partial E^{\left(x, p_{x}\right)}}{\partial a_{0}} .
$$

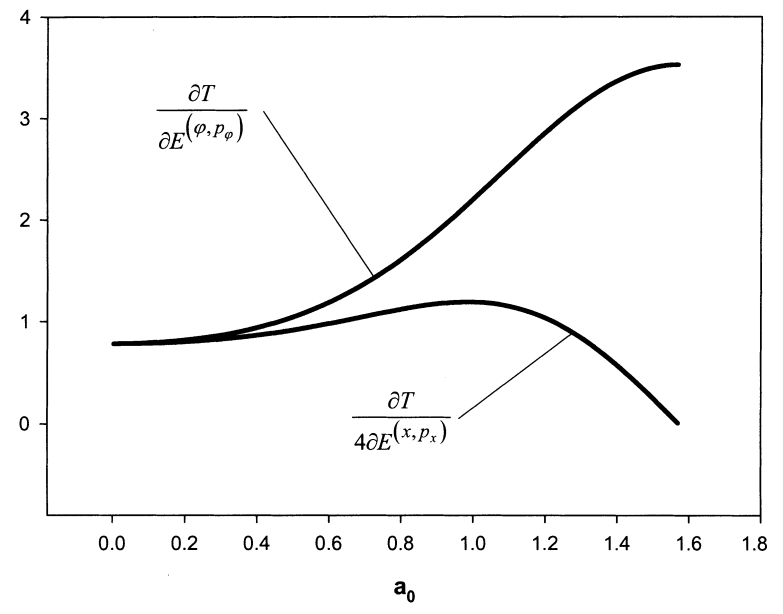

Fig. 4. The rates of change of the oscillation period $T$ with the energy $E^{\left(\varphi, p_{\varphi}\right)}$ [see (2), (6) and (43)] and the energy $4 E^{\left(x, p_{x}\right)}$ [see (16), (22), and (43a)] plotted as functions of $a_{0}$.

\section{Summary}

We obtained a Hamiltonian which is symmetrical in the canonically conjugated coordinates of position and momentum, $x$ and $p_{x}$. The Hamiltonian leads - for both kinds of coordinates - to equations of motion which are identical to that characteristic for the angular coordinate of $\varphi$ of the mathematical pendulum. The symmetry of the Hamiltonian becomes a symmetry of the phase-space plots of $p_{x}$ versus $x$ absent in traditional plots of the momentum $p_{\varphi}$ versus $\varphi$. The new Hamiltonian can easily be referred to the exact pendulum Hamiltonian, and the derived equations of motion can easily be solved with the aid of Fourier series.

The action functions of both Hamiltonians are calculated and mutually compared. Similar calculations are done for the parameter of non-linearity which characterizes the two pendulum systems, and the rate of change of the oscillation period obtained with the change of the pendulum energy is also computed.

Plots of the formulae (43) and (43a) presented as functions of $a_{0}$ are given in Figure 4. 
[1] S. J. Liao, J. Appl. Mech. 59, 970 (1992).

[2] Y. Minesaki and Y. Nakamura, Phys. Lett A 250, 300 (1998).

[3] G. Rangarajan, J. Phys. A Math. Gen. 31, 3649 (1998)

[4] E. A. Butcher and S. C. Sinha, Nonlinear Dyn. 17, 1 (1998).

[5] J. Awrejcewicz and I. V. Andrianov, Z. Angew. Math. Mech. 80, 265 (2000).

[6] C. Erkal, Europ. J. Phys. 21, 377 (2000).

[7] A. J. Dragt, F. Neri, and G Rangarajan, Phys. Rev. A 45, 2572 (1992).

[8] T. M. Janaki and G. Rangarajan, Phys. Rev. E 59, 4577 (1999).

[9] W. Dittrich and M. Reuter, Classical and Quantum Dynamics, Springer-Verlag, Berlin 1992.
[10] A. Sommerfeld, Mechanik, 4th ed., Akademische Verlagsgesellschaft, Leipzig 1949.

[11] B. V. Chirikov, Phys. Rep. Phys. Lett. 52, 263 (1979).

[12] U. Fischer and W. Stephan, Mechanische Schwingungen, 2nd ed., VEB, Leipzig 1984.

[13] M. Tabor, Chaos and Integrability in Nonlinear Dynamics, John Wiley, New York 1989.

[14] J. B. Marion and S. T. Thornton, Classical Dynamics of Particles and Systems, 4th ed., Saunders College, Fort Worth 1995.

[15] A. J. Lichtenberg and M. A. Liebermann, Regular and Stochastic Motion, Springer-Verlag, New York 1981.

[16] H. B. Dwight, Tables of Integrals and other Mathematical Data, 4th ed., MacMillan, New York 1961.

[17] W. Gröbner and N. Hofreiter, Integraltafel, Vol. 2, 2nd ed., Springer-Verlag, Wien 1958. 